\title{
Propiedades Psicométricas de la Versión Argentina de la Escala de Personalidad Proactiva
}

\author{
Solana Salessi ${ }^{1}$ \\ Alicia Omar ${ }^{2}$ \\ ${ }^{1}$ Universidad Nacional de Rafaela, Rafaela, Santa Fe, Argentina \\ ${ }^{2}$ Universidad Nacional de San Luis, Rosario, Santa Fe, Argentina
}

\begin{abstract}
Resumen
La personalidad proactiva es una tendencia relativamente estable a generar cambios en el ambiente para mejorarlo. El objetivo de este estudio fue traducir, adaptar y validar la Escala de Personalidad Proactiva de Bateman y Crant en Argentina. Se empleó un diseño empírico-instrumental transversal sobre una muestra no probabilística de 465 trabajadores $\left(54 \%\right.$ mujeres; $X_{\text {edad }}=$ 34,5 años; $\left.D T_{\text {edad }}=12,94\right)$. Análisis factoriales exploratorios y confirmatorios indicaron la adecuación de un modelo unifactorial con seis variables observables $\left(\mathrm{SB} \chi^{2}=2,01 ; \mathrm{GFI}=0,98 ; \mathrm{CFI}=0,95 ; \mathrm{RMSEA}=0,04, \mathrm{AIC}=42,03 ; \omega=0,82 ; \mathrm{CR}=0,80\right)$. $\mathrm{Las}$ correlaciones positivas entre personalidad proactiva, comportamientos proactivos, satisfacción e implicación laboral indicaron apropiada evidencia de validez de relación con variables externas. Los resultados obtenidos muestran que la versión argentina del instrumento presenta adecuadas propiedades psicométricas para el contexto laboral.
\end{abstract}

Palabras clave: proactividad; personalidad; validación estadística

Psychometric Properties of the Argentine Version of Proactive Personality Scale

\begin{abstract}
The proactive personality is a relatively stable tendency to generate changes in the environment to improve it. The aim of this study was to translate, adapt and validate the Proactive Personality Scale of Bateman and Crant in Argentina. A cross-sectional empirical-instrumental design was used on a non-probabilistic sample of 465 workers $\left(54 \%\right.$ women, $\mathrm{M}_{\text {age }}=34,5$ years, $\mathrm{SD}_{\text {age }}$ $=12,94)$. Exploratory and confirmatory factorial analyses indicated the adequacy of a unifactorial model with six observable variables $\left(\mathrm{SB} \chi^{2}=2,01 ; \mathrm{GFI}=0,98 ; \mathrm{CFI}=0,95 ; \mathrm{RMSEA}=0,04 ; \mathrm{AIC}=42,03 ; \omega=0,82 ; \mathrm{CR}=0,80\right)$. The positive correlations between proactive personality, proactive behaviours, job satisfaction and job involvement indicated appropriate relationship validity with external variables. These results show that the Argentine version of the instrument has adequate psychometric properties in the job context.
\end{abstract}

Keywords: proactivity; personality; statistical validity

Propriedades Psicométricas da Versão Argentina da Escala de Personalidade Proativa

\begin{abstract}
Resumo
A personalidade proativa é uma tendência relativamente estável de gerar mudanças no meio ambiente para melhorá-lo. O objetivo deste estudo foi traduzir, adaptar e validar a Escala de Personalidade Proativa de Bateman e Crant para a Argentina. Um desenho empírico-instrumental transversal foi utilizado em uma amostra não probabilística de 465 trabalhadores (54\% mulheres, $\mathrm{M}_{\text {idade }}=34,5$ anos, $\left.\mathrm{DP}_{\text {idade }}=12,94\right)$. Análises fatoriais exploratórias e confirmatória indicaram a adequação de um modelo unifatorial com seis variáveis observáveis $\left(\mathrm{SB} \chi^{2}=2,01\right.$; $\mathrm{GFI}=0,98 ; \mathrm{CFI}=0,95$; $\mathrm{RMSEA}=0,04 ; \mathrm{AIC}=42,03 ; \omega=0,82$; $\mathrm{CR}=$ $0,80)$. As correlações positivas entre personalidade proativa, comport amentos proativos, satisfação no trabalho e envolvimento no trabalho indicaram apropriada evidência de validade na relação com variáveis externas. Esses resultados mostram que a versão argentina do instrumento possui propriedades psicométricas adequadas para o contexto do trabalho.

Palavras-chave: proatividade; personalidade; validade estatística
\end{abstract}

Hacia finales del siglo XX los estudios sobre comportamiento organizacional cambiaron radicalmente sus concepciones sobre motivación y desempeño. En contraposición a las perspectivas tradicionales inspiradas en los principios del conductismo ortodoxo, la psicología organizacional contemporánea otorgó un rol activo al trabajador; quien, a partir de entonces, comenzó a ser considerado hacedor de cambios y trasformaciones (Parker \& Bindl, 2017). Como corolario de esta nueva concepción, comenzaron a proliferar estudios focalizados en desentrañar cómo los trabajadores influyen sobre su propio trabajo e incluso, sobre los resultados organizacionales a través de, por ejemplo, el rediseño de sus tareas y roles, la negociación de sus trayectorias profesionales, la innovación de métodos y procedimientos, la creación y expansión de sus redes sociales, etc. (Grant \& Ashford, 2008). A partir de los cimientos forjados por tales estudios fue conformándose una nueva línea de investigación, recogida en la literatura bajo el nombre de proactividad.

Bajo la etiqueta proactividad se hace referencia a las conductas auto-motivadas y orientadas al 
cambio que pretenden influir en el ambiente con el fin de lograr ciertos objetivos individuales, grupales u organizacionales (Bateman \& Crant, 1993; Parker \& Collins, 2010; Tornau \& Frese, 2013). Si bien podría presuponerse que la proactividad se reduce exclusivamente a conductas extra-rol, los especialistas (Belschak \& Den Hartog, 2017; Grant \& Ashford, 2008) coinciden en señalar que todos los tipos de desempeño (sean ejecuciones inherentes al rol o acciones que trascienden sus requerimientos formales) pueden ser más o menos proactivos. En efecto, las notas distintivas de la proactividad pueden reducirse, esencialmente, a tres aspectos: (a) que se trate de comportamientos impulsados por motivaciones intrínsecas (antes conductas coaccionadas); (b) que tales comportamientos se encuentren centrados en el futuro (es decir, relacionados a la anticipación de oportunidades y a la prevención de problemas) y; (c) que tales acciones estén orientadas a producir cambios en uno mismo, en el entorno o en los demás (Parker \& Bindl, 2017).

La investigación desarrollada durante los últimos treinta años proporciona numerosas evidencias sobre las diferentes formas que puede asumir la proactividad. Desde la óptica de algunos especialistas (Belschak \& Den Hartog, 2017; Grant \& Ashford, 2008) estas pueden ser subsumidas en las categorías de proactividad pro-individual (centrada en mejorar el propio desempeño); proactividad prosocial (direccionada a potenciar el funcionamiento del equipo) y; proactividad pro-organizacional (destinada a contribuir a que la organización logre sus metas). En tanto que, otros autores (Parker \& Collins, 2010) proponen una clasificación que distingue las categorías de: (a) comportamientos proactivos de ajuste persona-ambiente (tendientes a lograr una mayor compatibilidad entre las propias habilidades, valores, intereses y las expectativas y requerimientos del puesto y de la organización); (b) comportamientos laborales proactivos (orientados a introducir mejoras en el ambiente laboral inmediato) y; (c) comportamientos estratégicos proactivos (direccionados a promover la adaptación de la organización al medio más amplio del cual forma parte).

En contraposición a un enfoque que concibe a la proactividad en términos de comportamientos concretos, otros expertos abogan por una perspectiva disposicional. En este sentido, el concepto de personalidad proactiva (Bateman \& Crant, 1993) identifica las diferencias interpersonales en la predisposición a influir sobre el entorno en busca de cambios constructivos. La personalidad proactiva constituye una tendencia relativamente estable a generar cambios en el ambiente, en lugar de verse constreñido a actuar por presiones o requerimientos externos. Vale decir que, las personas con personalidades proactivas se destacan por su inclinación a identificar oportunidades de mejora, mostrar iniciativa, enfrentar los acontecimientos activamente, comprometerse más allá de lo estrictamente requerido y asumir con optimismo los desafíos. Tales características han sido empíricamente demostradas, habida cuenta que numerosas evidencias señalan que la personalidad proactiva es un importante predictor del desempeño, la satisfacción laboral, el compromiso organizacional y los comportamientos de ciudadanía (Crant, Hu, \& Jiang, 2017; Spitzmuller, Sin, Howe \& Fatimah, 2015; Thomas, Whitman, \& Viswesvaran, 2010).

La concepción de Bateman y Crant (1993) describe a la proactividad como una tendencia estable de la personalidad asumiendo, en consecuencia, una perspectiva de rasgo (Crant et al., 2017). Sin embargo, más allá de los hallazgos preliminares comunicados por los propios autores sobre la estabilidad de la proactividad en el tiempo (similar a la que cabría esperar para una disposición); pocos estudios se han expedido sobre esta cuestión. Algunas investigaciones recientes provenientes del campo de la genética conductual proveen cierta evidencia consistente con esta conceptualización. Así, por ejemplo, un estudio efectuado sobre una muestra de 1000 gemelos en Estados Unidos (Liu, 2013) ha encontrado que el $42 \%$ de la varianza de la personalidad proactiva es atribuible a diferencias genéticas. Al respecto, cabe destacar que se ha señalado (Bouchard, 2013) que, usualmente, el rango en el cual oscila la influencia de los genes en otros rasgos de personalidad varía entre el $40 \%$ y el $60 \%$. En la misma línea, otra investigación desarrollada con dos muestras independientes en China y en Estados Unidos (Liu et al., 2015) ha demostrado que cierto marcador genético (DAT1) está asociado a la personalidad proactiva. Tales hallazgos, aunque exiguos, resultan alentadores para quienes defienden la naturaleza disposicional de la proactividad.

Sin embargo, otros autores (Belschak \& Den Hartog, 2017; Grant \& Ashford, 2008) argumentan que el enfoque disposicional ofrece muy poca información respecto a qué comportamientos deberían ser clasificados como proactivos. Asimismo, cuestionan que este enfoque se focalice en la personalidad de los individuos que tienden a comportarse proactivamente, en lugar de centrarse en las conductas en sí mismas. En un intento de conciliar ambas posturas extremas, recientemente Crant et al. (2017) han admitido que la personalidad proactiva 
es sólo uno de los componentes de una constelación más amplia de constructos relacionados a la proactividad, tales como, comportamientos proactivos, comportamiento innovador e iniciativa personal, coincidiendo así con las conclusiones de otros especialistas (Parker \& Collins, 2010; Thomas et al., 2010; Tornau \& Frese, 2013).

Como corolario de su conceptualización, Bateman y Crant (1993) desarrollaron un instrumento de medida que permitiera operacionalizar esa tendencia: la Escala de Personalidad Proactiva. La validación inicial fue realizada en Estados Unidos sobre 546 participantes distribuidos en tres muestras diferentes. Dos de ellas estuvieron integradas por estudiantes universitarios enrolados en dos seminarios de comportamiento organizacional (Muestra 1: $n=282$; Muestra 2: $n=130$ ) y; la tercera, por alumnos de un posgrado en negocios con experiencia laboral (Muestra 3: $n=134)$. De un conjunto inicial de 47 ítems, los autores seleccionaron 27 reactivos para conformar la versión preliminar del instrumento. Sobre los datos proporcionados por la primera muestra se realizaron análisis factoriales exploratorios; los que arrojaron una solución unidimensional conformada por 17 ítems con alta confiabilidad $(\alpha=0,89)$. Idéntica solución fue encontrada en las dos muestras restantes; demostrándose, además, una adecuada confiabilidad test-retest a lo largo de un período de tres meses $(\alpha=0,79)$.

La Escala de Personalidad Proactiva (Bateman \& Crant, 1993) ha sido validada en otros contextos culturales tales como, por ejemplo, China (Zhou \& Shi, 2009), Turquía (Erdongan \& Bauer, 2005), Italia (Trifiletti, Capozza, Pasin, \& Falvo, 2009) y Bélgica (Pringels \& Claes, 2001). Sin embargo, hasta el momento no se dispone de una versión validada para la Argentina. Frente a este panorama, el presente estudio se propuso cubrir ese vacío empírico-instrumental persiguiendo, en consecuencia, un objetivo doble. Por un lado, traducir, adaptar y validar la Escala de Personalidad Proactiva (Bateman \& Crant, 1993) para su empleo con trabajadores argentinos. Por otro lado, analizar las propiedades psicométricas del instrumento, a través de la verificación de la consistencia interna y la búsqueda de evidencia de validez, mediante el análisis de la estructura interna y de su relación con variables externas que, a partir de la revisión de la literatura especializada refuerzan la hipótesis de la relación entre los comportamientos proactivos, la satisfacción laboral y la implicación con el trabajo (Belschak, Den Hartog, \& Fay, 2010; Bergeron, Schroeder, \& Martinez, 2014; Salessi \& Omar, 2018a; 2018b; Spitzmuller et al., 2015; Thomas et al., 2010).

\section{Método}

\section{Participantes}

Para realizar la traducción de los ítems se integró una muestra de seis jueces expertos, traductores profesionales de inglés $\left(\mathrm{M}_{\text {edad }}=32\right.$ años; $\left.D T=8,14\right)$. Para el análisis de la validez de contenido participaron, a su vez, otros tres jueces; doctores en psicología y especialistas en psicología del trabajo y las organizaciones $\left(\mathrm{M}_{\text {edad }}=54\right.$ años; $D T=12,93$ ).

Para llevar a cabo el estudio piloto se conformó una muestra no probabilística seleccionada por disponibilidad con 62 personas. Los participantes cursaban estudios de posgrado en administración de empresas y en dirección estratégica de recursos humanos en una universidad privada y en una universidad pública argentina, respectivamente. El 56\% de la muestra eran varones. La edad promedio fue de 33 años $(D T=$ $6,24)$. El $89 \%$ de los participantes del estudio trabajaban en diversas organizaciones industriales y de servicios localizadas en la ciudad de Rosario y Gran Rosario, siendo su antigüedad laboral media de 4,14 años $(D T=3,81)$.

Para el estudio principal se integró una nueva muestra por disponibilidad de 523 participantes, de los cuales fueron descartados 46 casos incompletos. La muestra definitiva quedó conformada por 477 trabajadores (54,5\% mujeres) de diversas organizaciones argentinas públicas y privadas localizadas en el centro-sur de la provincia de Santa Fe y norte de la provincia de Buenos Aires. El promedio de edad fue de 34,5 años (DT = 12,94). La antigüedad laboral media fue de 8,6 años ( $D T$ $=10,21)$. El 84,5\% de la muestra tenía estudios superiores terciarios y/ o universitarios. El 55,2\% trabajaba en el sector privado. La distribución por rubros de actividad fue la siguiente: $32,2 \%$ industria; $30,5 \%$ comercio y servicios; $25,3 \%$ salud; $12 \%$ educación.

\section{Instrumentos}

Escala de Personalidad Proactiva. El instrumento desarrollado por Bateman y Crant (1993) evalúa la personalidad proactiva, definida como una tendencia estable a generar cambios en el ambiente. La escala está integrada por 17 ítems (ej.: "siempre estoy buscando formas de hacer mejor las cosas") valorados sobre una escala tipo Likert de 5 puntos ( $1=$ totalmente en desacuerdo; $5=$ totalmente de acuerdo). Evidencias de estructura interna (Claes, Beheydt, \& Lemmens, 2005) sustentan la idoneidad de un modelo unifactorial $\left(\mathrm{SB} \chi^{2} / \mathrm{gl}=2.73\right.$; GFI= .96 ; $\mathrm{CFI}=.98$; $\mathrm{RMSEA}=.04)$. 
Escala de Comportamientos Proactivos. El instrumento desarrollado por Belschak y Den Hartog (2010) evalúa los comportamientos proactivos, entendidos como conductas auto-motivadas orientadas al cambio constructivo que pretenden influir sobre la organización, sobre los demás, o sobre uno mismo. La versión utilizada en este estudio (Salessi \& Omarm, 2018a) está integrada por 10 ítems presentados en formato Likert de 5 puntos ( $1=$ totalmente en desacuerdo; $5=$ totalmente de acuerdo). Los reactivos se distribuyen a lo largo de tres factores denominados: comportamientos proactivos orientados a la organización (3 ítems; ej: "sugiero ideas frente a los problemas que afronta mi organización"); comportamientos proactivos orientados a los demás (4 ítems; ej: "ayudo a mis compañeros para que desarrollen e implementen nuevas ideas") y; comportamientos proactivos orientados a uno mismo (3 ítems; ej: "encuentro nuevas maneras de realizar mis tareas y ser más efectivo"). Evidencias de estructura interna (Salessi \& Omar, 2018a) indican la adecuación de un modelo trifactorial oblicuo integrado por 10 variables observables $\left(\mathrm{SB} \chi^{2} / \mathrm{gl}=1.79 ; \mathrm{GFI}=.95 ; \mathrm{CFI}=\right.$ .94; RMSEA= .03).

Cuestionario de Implicación en el Trabajo. El instrumento desarrollado por Kanungo (1992) evalúa la implicación laboral definida como el grado en que una persona está cognitiva y emocionalmente comprometida e involucrada con su trabajo actual. La versión utilizada en este estudio (Salessi \& Omar, 2018b) está integrada por 9 ítems (ej.: “mi trabajo ocupa un lugar irremplazable en mi vida") valorados sobre una escala tipo Likert de 5 puntos ( $1=$ totalmente en desacuerdo; $5=$ totalmente de acuerdo). Evidencias de estructura interna (Salessi \& Omar, 2018b) demuestran la adecuación de un modelo unifactorial con 9 variables observables $\left(\mathrm{SB} \chi_{\mathrm{gl}}^{2 /}=1.81, \mathrm{GFI}=.91, \mathrm{CFI}=\right.$ .90, RMSEA $=.03$ )

Escala de Satisfacción Laboral Genérica. El instrumento desarrollado por Mac Donald y Mac Intyre (1997) evalúa la satisfacción laboral entendida como una actitud general; vale decir, un conjunto de respuestas cognitivas y afectivas frente la situación laboral. La versión utilizada en este estudio está integrada por 7 ítems (ej.: "en mi trabajo puedo aplicar todas mis capacidades y habilidades") valorados sobre una escala tipo Likert de 5 puntos $(1=$ totalmente en desacuerdo; $5=$ totalmente de acuerdo). Evidencias de estructura interna (Salessi \& Omar, 2016) indican un modelo unifactorial integrado por 7 variables observables $\left(\mathrm{SB} \chi^{2} /_{\mathrm{gl}}=1.12\right.$; $\mathrm{GFI}=.93$; CFI $=.94$; $\mathrm{RMSEA}=.03$ ).

\section{Procedimiento}

Adaptación lingüística. Se tradujeron los ítems siguiendo un procedimiento iterativo de traducción y re-traducción. En primer lugar, dos de los jueces tradujeron los ítems desde su idioma original (inglés) al idioma de la población objetivo (español-argentino) atendiendo a aspectos lingüísticos y culturales. Seguidamente, otros dos jueces retradujeron los ítems desde el español al inglés. Finalmente, los dos expertos restantes compararon "a ciegas" las dos formas del instrumento. Los profesionales concluyeron que se había mantenido inalterada la concordancia entre cada ítem original y el traducido al español.

Análisis de validez de contenido. A partir del análisis de la literatura especializada (Crant et al., 2017; Praker \& Bindl, 2017; Spitzmuller et al., 2015; Thomas et al., 2010) y del juicio de los tres especialistas consultados se estableció que el constructo personalidad proactividad era empíricamente distinguible de otros constructos con los cuales existe cierto solapamiento conceptual (por ejemplo, iniciativa personal, toma de control y autoeficacia para la amplitud del rol). De particular relevancia en esta etapa fue la investigación de Claes et al. (2005), que permitió concluir que el significado del constructo era equivalente en la cultura en la que el instrumento fue desarrollado y en la cultura donde el mismo sería aplicado.

Análisis de equivalencia operacional. La posibilidad de mantener estables las características operativas durante la aplicación del instrumento (por ejemplo, mediante la utilización del mismo formato de respuesta, el uso de instrucciones similares a las originales, etc) se examinó a partir de los resultados de un estudio piloto. Para esto, se desarrolló la recolección de los datos en las instalaciones de cada una de las dos universidades participantes, previa autorización de las autoridades correspondientes. La administración se realizó en los días habituales de cursado y en los horarios regulares de clases. La aplicación del protocolo fue realizada de manera colectiva en las respectivas aulas de cursado. En todos los casos, se explicaron los objetivos y la naturaleza del estudio, solicitando la colaboración voluntaria y anónima de los alumnos, y enfatizando la confidencialidad de la información recolectada. La administración de la escala llevó alrededor de 15 minutos. Una vez concluida, se habilitó un espacio para que los participantes pudieran opinar del instrumento; principalmente, en lo referente a posibles ambigüedades de sentido, claridad de la redacción, tiempo requerido para completarla. Los participantes del estudio piloto señalaron que las 
instrucciones para realizar la tarea estaban claramente redactadas; que no tuvieron inconvenientes para comprender el contenido de los ítems, y que la escala tipo Likert utilizada para responder no generaba dificultades.

Análisis de propiedades psicométricas. Para analizar la estructura interna y obtener evidencias de validez de la escala adaptada se efectuó una nueva recolección de datos. La misma se desarrolló en el seno de las organizaciones que aceptaron participar de la investigación, en días y horarios previamente pautados con las autoridades, y en los lugares físicos que las mismas dispusieron a tal efecto. La participación de los trabajadores fue voluntaria, anónima y confidencial. No se brindaron incentivos de ningún tipo. En todos los casos, los participantes completaron de manera individual un cuadernillo conteniendo en su primera hoja el objetivo del estudio y las instrucciones para responder; en la segunda hoja el formulario de consentimiento informado y; en las restantes páginas los ítems, distribuidos aleatoriamente, correspondientes a las escalas de personalidad proactiva, comportamientos proactivos, implicación con el trabajo y satisfacción laboral. La administración de las técnicas estuvo a cargo de personal entrenado a tal efecto. El tiempo aproximado que demandó completar el cuadernillo fue de 30 minutos.

\section{Estrategia de análisis de los datos}

Los diversos análisis estadísticos que se describen a continuación fueron realizados con el auxilio de los programas informáticos SPSS (versión 23.0), Factor (versión 10.5) y EQS (versión 6.3), según corresponda.

Análisis exploratorios preeliminares. La matriz de datos fue analizada con miras a detectar la presencia de valores perdidos y puntuaciones extremas. Se identificaron los casos atípicos mediante el cálculo de puntuaciones $Z$ y las distancias de Mahalanobis al cuadrado $\left(D^{2}\right)$ para cada variable. Valores de asimetría y curtosis inferiores a $\pm 2,00 \mathrm{y}$, correlaciones positivas superiores a 0,30 se consideraron adecuados (Tabachnick \& Fidell, 2013).

Evidencias de estructura interna. Sobre una mitad de la muestra se ejecutó un análisis factorial exploratorio (AFE), previa obtención de los índices de adecuación muestral (pruebas de Kaiser-Meyer-Olkin y de Esfericidad de Bartlett). Por la naturaleza ordinal de los datos se utilizó la matriz de correlaciones policóricas (Hoffmann, Stover, De la Iglesia, \& Fernández-Liporace, 2013). Para determinar el número de factores se utilizó el método de estimación DWLS (Diagonally Weighted Squares), recomendado por especialistas (Li, 2016) cuando el tamaño muestral es de alrededor de 200 participantes, y se aplicó rotación Oblimin. Asimismo, se realizó un análisis paralelo optimizado, extrayendo aleatoriamente 500 sub-matrices e implementando el análisis de rango mínimo. Complementariamente, se efectuó un análisis con el método Hull (Timmerman \& Lorenzo-Seva, 2011). El criterio para la selección de los ítems fue que pesaran 0,40 o más sobre el factor, y que no saturaran sobre más de un factor al mismo tiempo (Lloret-Segura, Ferreres, Hernández, \& Tomás, 2017).

Sobre la otra mitad de la muestra se llevó a cabo un análisis factorial confirmatorio (AFC) siguiendo la estrategia de modelos rivales, habida cuenta que la literatura científica indica la idoneidad de otros modelos de medida. Por ende, el modelo derivado del AFE (Modelo A) fue contrastado con tres modelos unifactoriales rivales: (1) modelo B conformado por 10 ítems como indicadores observables (Seibert, Crant, \& Kraimer, 1999); (2) modelo $C$ conformado por 6 ítems como indicadores observables (Parker, 1998) y; (3) modelo D conformado por 4 ítems como indicadores observables (Parker \& Sprigg, 1999). Para estimar el grado de ajuste del modelo se utilizó el método de máxima verosimilitud con la corrección robusta de Satorra-Bentler (SB), dada la naturaleza ordinal de los datos (Bentler, 2006). Para evaluar la bondad de ajuste se analizó el índice $\mathrm{SB} \chi^{2}$ sobre los grados de libertad $\left(\mathrm{SB} \chi^{2} / \mathrm{gl}\right)$ fuera inferior a 3, el índice de bondad de ajuste (GFI) y el índice de ajuste comparativo (CFI) alcanzaran valores iguales o superiores a 0,90 , el error cuadrático medio de aproximación (RMSEA) fuera inferior a 0,05, y el Criterio de Información de Akaike (AIC).

Evidencias de confiabilidad. La confiabilidad y consistencia se estableció a partir del computó del coeficiente de confiabilidad compuesta (CR, del inglés Composite Reliability), y del coeficiente Omega de McDonald.

Evidencias de validez de relación. Se obtuvo evidencia de validez de relación con variables externas mediante el cómputo del coeficiente de rho Spearman $(r)$ para las correlaciones entre los constructos evaluados.

\section{Resultados}

\section{Análisis exploratorios preeliminares}

El porcentaje de datos perdidos en cada ítem no superó el 5\%. Los valores faltantes fueron reemplazados mediante el método de Estimación-Maximización 
(Tabachnick \& Fidell, 2013). Se detectaron 12 casos atípicos, 9 de los cuales satisfacían la condición para considerarse atípicos multivariados. Los mismos fueron excluidos de la matriz, quedando la muestra final constituida por 465 participantes. No se observaron problemas de asimetría y curtosis, y las correlaciones ítem-total fueron todas positivas. La descripción de los ítems se presenta en la Tabla 1.

\section{Evidencias de estructura interna}

Análisis factorial exploratorio. Atendiendo a la recomendación de contar con un mínimo de 200 observaciones para asegurar que la solución factorial fuera estable y generalizable (Lloret-Segura et al., 2017) se seleccionaron en forma aleatoria simple 233 casos. La matriz de datos fue considerada factorizable (Test de Esfericidad de Bartlett: $\chi_{(136 ; 233)}^{2}=1167,47 ; p$ $=0,000$; Índice de Adecuación Muestral de Kaiser-Meyer-Olkin $=0,80)$. La varianza explicada basada en los eigenvalues indicó la pertinencia de retener sólo un factor, el cual explicó el 58,40\%. Del mismo modo, el análisis paralelo optimizado sugirió un factor con autovalor superior a su equivalente en la matriz de datos aleatorios (Autovalor real $=6,86$; Media autovalor aleatorio $=1,47$; Percentil 95 de autovalor aleatorio $=1,53$ ). Idéntica solución arrojó el método Hull, que indicó la retención de un factor con valor propio. En la Tabla 1 se informa la matriz factorial con las saturaciones de cada ítem.

Análisis factorial confirmatorio. Sobre la otra mitad de la muestra $(\mathrm{n}=232)$ se llevó a cabo un análisis factorial confirmatorio. Los resultados obtenidos para los diversos modelos contrastados se presentan en la Tabla 2.

La inspección de la Tabla 2 muestra que los índices obtenidos para el modelo $\mathrm{A}$ indicaron un ajuste deficiente. El examen de las cargas factoriales indicó que cuatro ítems (ítem 2: "me siento impulsado a hacer una diferencia en la sociedad y, tal vez, en el mundo"; ítem 3: "tiendo a dejar que otros tomen la iniciativa de comenzar nuevos proyectos"; ítem 13: "me gusta el desafío de modificar el modo cómo las cosas son para mejorarlas"; ítem 17: "si alguien está en problemas, lo trato de ayudar de cualquier manera posible") presentaban saturaciones inferiores a los valores usualmente considerados satisfactorios $(\lambda \geq 0,50)$. Frente a este panorama, se decidió eliminarlos obteniendo una ganancia en el ajuste del modelo; el cual, sin embargo, continuó siendo inadecuado (Modelo $\mathrm{A}_{(1) \text { re-especificado }}$ ). La inspección de los índices de modificación indicó que la correlación entre los residuos pertenecientes al par conformado por los ítems 5 ("disfruto enfrentar y superar los obstáculos que se anteponen a mis ideas") y 9 ("me gusta defender mis ideas cuando los demás se oponen”) produciría una mejoría en el modelo. Dado que residuos altamente correlacionados indican redundancia debido a la superposición de contenidos (Brown, 2015), se decidió eliminar el ítem que presentó un menor peso de regresión estandarizado (ítem 5). Si bien el modelo re-especificado presentó un mejor ajuste, éste no fue óptimo (Modelo $\left.\mathrm{A}_{(2) \text { re-especificado }}\right)$.

$\mathrm{El}$ análisis del modelo $\mathrm{B}$ muestra un ajuste aceptable. No obstante, los índices de modificación indicaron que la correlación entre los residuos pertenecientes a los pares conformados por los ítems 1 ("busco constantemente cómo mejorar mi vida") y 11 ("siempre estoy buscando formas de hacer mejor las cosas") y; por los ítems 10 ("soy muy bueno identificando oportunidades") y 16 ("me doy cuenta de las oportunidades antes que los demás") produciría una ganancia en el modelo. Frente a la superposición de contenido, se optó por eliminar los ítems 1 y 16 dada su menor carga factorial. El modelo $\mathrm{B}_{(1)}$ re-especificado presentó un ajuste apropiado, pero no óptimo.

En cuanto al modelo C, los índices obtenidos para este modelo indicaron un ajuste satisfactorio y una confiabilidad adecuada. En tanto que, el modelo D exhibió un ajuste adecuado, pero no óptimo, y una confiabilidad preliminar que no alcanzó los mínimos recomendados por la literatura científica.

Por ende, de la comparación de los índices obtenidos para los diversos modelos contrastados surge que el modelo conformado por un factor latente y 6 indicadores como variables observables (Modelo C) fue el que presentó el mejor grado de ajuste, confiabilidad y consistencia interna. La Figura 1 ilustra el modelo de medida subyacente a la validación argentina de la Escala de Personalidad Proactiva.

\section{Evidencias de validez de relación}

La Tabla 3 muestra los estadísticos descriptivos y coeficientes de correlación entre las variables bajo estudio.

La inspección de la Tabla 3 muestra que la personalidad proactiva se asocia positivamente con los comportamientos proactivos, con la satisfacción laboral y con la implicación en el trabajo. Tales hallazgos aportan evidencias favorables respecto a la validez de relación de la escala aquí adaptada. 
Tabla 1.

Matriz factorial (AFE) y estadísticos descriptivos correspondiente a los 17 items de la Escala de Personalidad Proactiva (versión adaptada)

Contenido del ítem

\begin{tabular}{cccccc}
$\begin{array}{c}\text { Matriz } \\
\text { factorial }\end{array}$ & Media & DE & Asimetría & Curtosis & $\mathrm{r}_{\mathrm{i}}$-total \\
\hline 0,66 & 3,36 & 1,07 & 0,70 & 1,16 & 0,55 \\
0,41 & 2,68 & 0,98 & $-1,68$ & 1,54 & 0,39 \\
0,42 & 2,53 & 0,97 & $-1,17$ & $-1,21$ & 0,41
\end{tabular}

3. Tiendo a dejar que otros tomen la iniciativa de comenzar nuevos proyectos*

4. En cualquier contexto puedo lograr cambios positivos

5. Disfruto enfrentar y superar los obstáculos que se anteponen a mis ideas

6. Me gusta que mis ideas se conviertan en realidad

7. Si algo no me gusta trato de cambiarlo

0,70

3,15

1,02

$-0,31$

$-0,22$

0,67

0,68

3,19

1,11

0,92

0,78

0,65

8. Si creo en algo lo hago posible, aún contra todas las probabilidades

9. Me gusta defender mis ideas cuando los demás se oponen

10. Soy muy bueno identificando oportunidades

11. Siempre estoy buscando formas de hacer mejor las cosas

12. Si creo en una idea, ningún obstáculo me impedirá concretarla

13. Me gusta el desafío de modificar el modo cómo las cosas son para mejorarlas

$\begin{array}{llllll}0,62 & 3,42 & 1,04 & 0,72 & 0,67 & 0,53\end{array}$

$\begin{array}{llllll}0,81 & 3,89 & 1,01 & 0,42 & -0,34 & 0,72\end{array}$

$0,76 \quad 3,27$

1,13

0,35

$-0,25$

0,61

$\begin{array}{llllll}0,72 & 3,43 & 1,08 & 0,73 & -0,89 & 0,58\end{array}$

0,71

3,10

1,21

0,28

$-0,21$

0,60

0,84

3,76

1,18

$-0,35$

0,23

0,63

0,63

3,21

1,06

0,79

0,61

0,57

14. Cuando tengo un problema lo enfrento directamente

15. Soy muy bueno transformando los problemas en oportunidades

$\begin{array}{llllll}0,41 & 3,11 & 0,93 & -1,83 & -1,92 & 0,35 \\ 0,78 & 3,81 & 1,09 & 0,20 & 0,17 & 0,72 \\ 0,66 & 3,52 & 1,00 & -0,51 & 0,61 & 0,63 \\ 0,62 & 3,25 & 1,13 & 0,43 & -0,31 & 0,65 \\ 0,43 & 3,98 & 1,02 & 0,18 & 0,11 & 0,70\end{array}$

16. Me doy cuenta de las oportunidades antes que los demás

17. Si alguien está en problemas, trato de ayudarlo de cualquier modo posible

Nota. El asterisco indica ítems reversos

\section{Discusión}

El estudio instrumental aquí presentado tuvo un doble propósito. Por un lado, traducir, adaptar y validar la Escala de Personalidad Proactiva desarrollada por Bateman y Crant (1993) para la población argentina.
Por otro lado, analizar las propiedades psicométricas de esta versión. En lo que hace al primer objetivo, el criterio experto de los profesionales consultados indicó que la versión en español presenta equivalencia semántica, al conservar inalterado el sentido de cada ítem original en su respectiva traducción. El estudio piloto realizado 
Tabla 2.

Indices de ajuste y confiabilidad de los diversos modelos de medida alternativos contrastados

\begin{tabular}{|c|c|c|c|c|c|c|c|c|}
\hline Modelos de medida & $\mathrm{SB} \chi^{2 / g 1}$ & GFI & CFI & RMSEA & IC $95 \%$ & AIC & $\omega$ & CR \\
\hline Modelo A & 5,11 & 0,64 & 0,60 & 0,14 & {$[0,13 ; 0,15]$} & 375,49 & 0,66 & 0,64 \\
\hline Modelo $\mathrm{A}_{(1) \text { re-especificado }}$ & 4,61 & 0,67 & 0,63 & 0,13 & {$[0,13 ; 0,14]$} & 351,23 & 0,69 & 0,67 \\
\hline Modelo $A_{(2)}$ re-especificado & 4,14 & 0,70 & 0,69 & 0,11 & {$[0,10 ; 0,11]$} & 322,91 & 0,71 & 0,69 \\
\hline Modelo B & 3,85 & 0,80 & 0,83 & 0,10 & {$[0,09 ; 0,11]$} & 286,91 & 0,67 & 0,65 \\
\hline Modelo $B_{(1) \text { re-especificado }}$ & 3,49 & 0,87 & 0,87 & 0,08 & {$[0,07 ; 0,09]$} & 243,97 & 0,74 & 0,70 \\
\hline Modelo C & 2,01 & 0,98 & 0,94 & 0,04 & {$[0,03 ; 0,05]$} & 42,03 & 0,82 & 0,80 \\
\hline Modelo D & 3,35 & 0,89 & 0,90 & 0,07 & {$[0,06 ; 0,08]$} & 38,18 & 0,66 & 0,64 \\
\hline
\end{tabular}

Nota:

Modelo A: unifactorial con 17 indicadores como variables observables y sus respectivos errores de medida.

Modelo $\mathrm{A}_{(1) \text { re-especificado: }}$ unifactorial con 13 indicadores como variables observables y sus respectivos errores de medida.

Modelo $\mathrm{A}_{(2) \text { re-especificado: }}$ unifactorial con 12 indicadores como variables observables y sus respectivos errores de medida.

Modelo B: unifactorial con 10 indicadores como variables observables y sus respectivos errores de medida.

Modelo $\mathrm{B}_{(1) \text { re-especificado: }}$ unifactorial con 8 indicadores como variables observables y sus respectivos errores de medida.

Modelo C: unifactorial con 6 indicadores como variables observables y sus respectivos errores de medida.

Modelo D: unifactorial con 4 indicadores como variables observables y sus respectivos errores de medida.

determinó, a su vez, la equivalencia operacional, desde el momento que se replicaron las características operativas del estudio original (instrucciones, escala de respuesta tipo Likert, tiempo de administración, etc.) con resultados satisfactorios.

En cuanto al segundo objetivo, los análisis factoriales desarrollados apoyan tanto las conclusiones de Bateman y Crant (1993), como las de numerosas validaciones internacionales (Erdongan \& Bauer, 2005; Pringels \& Claes, 2001; Trifiletti et al., 2009; Zhou \& Shi, 2009) respecto a la estructura unidimensional de la escala. No obstante, a diferencia de esos estudios, los hallazgos de la presente investigación indican que el modelo de medida que mejor capta la esencia del constructo es aquél compuesto por un factor latente y 6 ítems como indicadores observables. El análisis de modelos rivales demostró que este modelo es el más parsimonioso y con mejor grado de ajuste a la matriz de varianzas y covarianzas. Dicho resultado armoniza con las conclusiones de Claes et al. (2005); quienes, a partir del estudio de tres muestras independientes pertenecientes a Bélgica, España y Finlandia, encontraron que, en comparación a la versión de 10 ítems, la escala integrada por seis reactivos presenta mejor ajuste, consistencia y confiabilidad test-retest en los tres países analizados.

Los resultados obtenidos en el presente estudio muestran que la versión argentina de la escala posee una aceptable consistencia interna y confiabilidad compuesta, comparable a la de la versión original y a la de las adaptaciones hasta el momento efectuadas (Claes et al. 2005; Erdongan \& Bauer, 2005; Pringels \& Claes, 2001; Trifiletti et al., 2009; Zhou \& Shi, 2009).

En lo que respecta a las evidencias de relación con variables externas, el análisis de correlaciones indica que la personalidad proactiva se asocia en la dirección esperada con los constructos evaluados. En este sentido, las asociaciones positivas observadas con los comportamientos proactivos no hacen sino replicar los resultados obtenidos por diversos estudios (Belschak et al., 2017; Crant, et al., 2017; Parker \& Bindl, 2017; Salessi \& Omar, 2018a); corroborando, una vez más, la vinculación entre la predisposición a controlar el ambiente (personalidad proactiva) y la posibilidad de manifestar acciones deliberadas orientadas al cambio (comportamientos proactivos).

A su vez, las correlaciones positivas con la satisfacción laboral se encuentran en sintonía con los hallazgos 


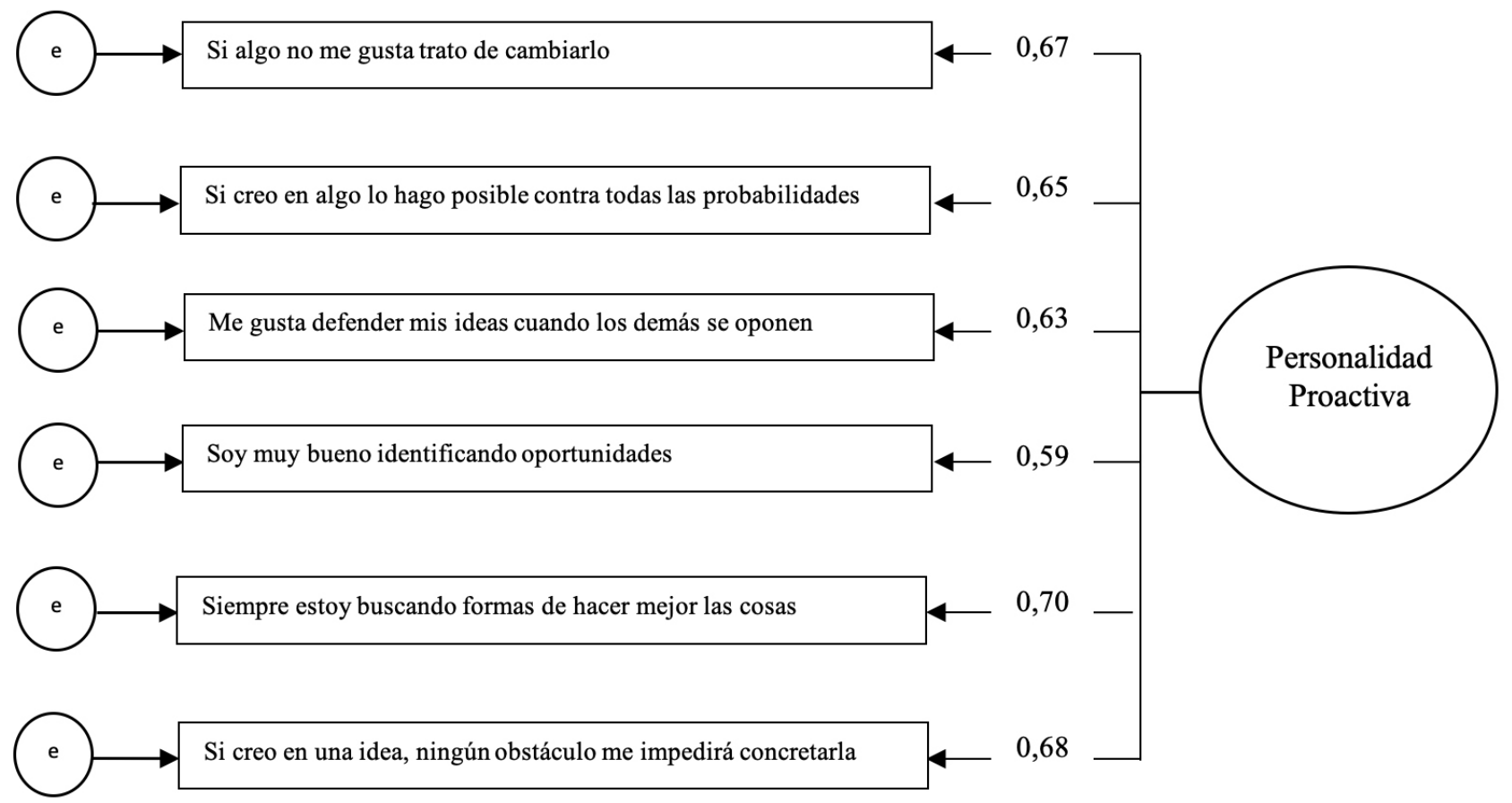

Figura 1. Modelo de medida correspondiente a la validación argentina de la Escala de Personalidad Proactiva.

Tabla 3.

Estadísticos descriptivos e indices de correlación de las variables bajo estudio

\begin{tabular}{lllllllll}
\hline & $\mathrm{M}$ & $\mathrm{DT}$ & 1 & 2 & 3 & 4 & 5 & 6 \\
\hline 1. Personalidad proactiva & 3,54 & 1,19 & - & $\mathbf{0 , 3 9}$ & $\mathbf{0 , 4 1}$ & $\mathbf{0 , 4 4}$ & $\mathbf{0 , 4 0}$ & $\mathbf{0 , 4 2}$ \\
2. CP organizacionales & 2,91 & 1,22 & & - & $\mathbf{0 , 4 6}$ & $\mathbf{0 , 4 3}$ & $\mathbf{0 , 4 1}$ & $\mathbf{0 , 3 8}$ \\
3. CP interpersonales & 3,35 & 1,36 & & & - & $\mathbf{0 , 4 2}$ & $\mathbf{0 , 3 1}$ & $\mathbf{0 , 2 6}$ \\
4. CP individuales & 3,56 & 1,27 & & & & - & $\mathbf{0 , 4 1}$ & $\mathbf{0 , 4 5}$ \\
5. Implicación laboral & 3,48 & 1,29 & & & & & - & $\mathbf{0 , 6 4}$ \\
6. Satisfacción laboral & 3,85 & 1,24 & & & & & & - \\
\hline
\end{tabular}

Nota. Negrita: $\boldsymbol{p}<\mathbf{0 , 0 1} . \mathrm{CP}=$ comportamientos proactivos

de numerosas investigaciones previas (Bergeron et al., 2014; Spitzmuller et al., 2015; Thomas et al., 2010; Tornau \& Frese, 2013), que indican que la personalidad proactividad se asocia a actitudes laborales positivas. En lo que respecta a la implicación laboral, si bien hasta la fecha son escasos los estudios que se han interesado por examinar sus vinculaciones directas, la evidencia disponible (Salessi \& Omar, en 2018b) muestra que los trabajadores proactivos se encuentran cognitiva y emocionalmente comprometidos con su trabajo, concibiéndolo como un dominio central en sus vidas y un aspecto crítico para su autoestima y autoimagen.
Entre las principales limitaciones de la investigación realizada hay que citar la representatividad de la muestra, que al haber sido seleccionada en forma no probabilística impide la generalización de los resultados a la población en su conjunto. Una segunda limitación podría estar vinculada con la validez externa del instrumento aquí presentado. En este sentido, como no se ha verificado la estabilidad a lo largo del tiempo sería recomendable que próximos estudios exploren la confiabilidad test-retest de esta versión. Del mismo modo, no haber analizado otros aspectos tales como la validez predictiva e incremental podría constituir una 
tercera limitación. Frente a este panorama, se alienta a que otras investigaciones examinen si las puntuaciones en esta escala constituyen predictores válidos y precisos de resultados relevantes para las organizaciones. Por último, cabe remarcar que como la escala evalúa los rasgos auto-percibidos, las respuestas podrían verse contaminadas tanto por la tendencia a aparecer mejor como por otros componentes subjetivos. Por lo tanto, sería importante en futuros estudios obtener información de otras fuentes (tales como la que pueden proporcionar compañeros y supervisores); así como, utilizar mecanismos de validez interna, tales como, por ejemplo, escalas de deseabilidad social.

\section{Referencias}

Bateman, T. S., \& Crant, J. M. (1993). The proactive component of organizational-behaviour: A measure and correlates. Journal of Organizational Behaviour, 14, 103-118. doi: 10.1002/job.4030140202

Belschak, F., \& Den Hartog, D. (2017). Foci of proactive behaviour. In S. Parker, \& U. Bindl, (Eds.), Proactivity at work: Making things happen in organizations (pp. 169-189). New York, NY: Routledge.

Belschak, F. D., Den Hartog, D. N., \& Fay (2010). Exploring positive, negative and context-dependent aspects of proactive behaviours at work. Journal of Occupational and Organizational Psychology, 83, 267273. doi: 10.1348/096317910x501143

Bentler, P. (2006). EQS 6 Structural Equations Program Manual. Los Angeles, CA: Multivariate Software Inc.

Bergeron, D., Schroeder, T., \& Martinez, H. (2014). Proactive personality at work: Seeing more to do and doing more? Journal of Business \& Psychology, 29, 71-86. doi: 10.1007/s10869-013-9298-5

Bouchard, T. (2013). Genetic influence on human psychological traits. In S. Downes, \& E. Machery (Eds.), Arguing about human nature: Contemporary debates (pp. 139-144). New York, NY, US: Routledge/ Taylor \& Francis Group.

Brown, T. A. (2015). Confirmatory factor analysis for applied research. New York, NY: Guilford Publications.

Claes, R., Beheydt, C., \& Lemmens, B. (2005). Unidimensionality of abbreviated Proactive Personality Scales across cultures. Applied
Psychology: An international review, 54, 476-489. doi: 10.1111/j.1464-05972005.00221x

Crant, M. Hu, J., \& Jiang, K. (2017). Proactive personality: A twenty-year review. In S. Parker, \& U. Bindl, (Eds.), Proactivity at work: Making things happen in organizations (pp. 194-225). New York, NY: Routledge

Erdongan, B., \& Bauer, T. (2005). Enhancing career benefits of employee proactive personality: The role of fit with jobs and organizations. Personnel Psychology, 58(4), 859-891. doi: 10.1111/j.1744-6570.200500772x

Grant, A. M., \& Ashford, S. J. (2008). The dynamics of proactivity at work. Research in Organizational Behaviour, 28, 3-34. doi: 10.1016/j.riob.2008.04.002

Hoffmann, A., Stover, J., De la Iglesia, G., \& Fernández-Liporace, M. (2013). Correlaciones policóricas y tetracóricas en estudios factoriales exploratorios y confirmatorios. Ciencias Psicológicas, 7, 151164. Recuperado de https://www.redalyc.org/ pdf/4595/459545415005.pdf

Kanungo, R. N. (1982). Measurement of job and work involvement. Journal of Applied Psychology, 67(3), 341-349. doi:10.1037/0021-9010.67.3.341

Li, C. (2016). Confirmatory factor analysis with ordinal data: Comparing robust maximum likelihood and diagonally weighted least squares. Behavior Research Methods, 48, 936-949. doi: 10.3758/ s13428-015-0619-7

Li, W. (2013). Proactive personality: Genetic influences in its relationships with career success and environmental impacts on its change. Unpublished doctoral dissertation. National University of Singapore.

Li, W., Wang, N., Arvey, R. D., Soong, R., Saw, S. M., \& Song, Z. (2015). A mixed blessing? Dual mediating mechanisms in the relationship between dopamine transporter gene DAT1 and leadership role occupancy. Leadership Quarterly, 26(5), 671-686. doi: 10.1016/j.leaqua.2014.12.005

Lloret, S., Ferreres, A., Hernández, A., \& Tomás, I. (2017). The exploratory factor analysis of items: Guided analysis based on empirical data and software. Anales de Psicología, 33(2), 417-432. doi: 10.6018/analesps.33.2.270211

Macdonald, S., \& MacIntyre, P. (1997). The generic job satisfaction scale. Employee Assistance Quarterly, 13, 1-16. doi: 10.1300/j022v13n02_01

Psico-USF, Bragança Paulista, v. 26, n. 2, p. 203-214, abr./jun. 2021 
Parker, S. (1998). Enhancing role breadth self-efficacy: The roles of job enrichment and other organizational interventions. Journal of Applied Psychology, 83, 835-852. doi: 10.1037//0021-9010.83.6.835

Parker, S., \& Bindl, U. (2017). Proactivity at work: A big picture perspective on a construct that matters. In S. K. Parker \& U. Bindl, U. (Eds.), Proactivity at work: Making things happen in organizations (pp. 1-20). New York, NY: Routledge.

Parker, S. K., \& Collins, C. (2010). Taking stock: Integrating and differentiating multiple proactive behaviours. Journal of Management, 36, 633-662. doi: 10.1177/0149206308321554

Parker, S., \& Sprigg, C. (1999). Minimizing strain and maximizing learning: The role of job demands, job control, and proactive personality. Journal of Applied Psychology, 84, 925-939. doi: 10.1037//0021-9010.84.6.925

Pringels, A., \& Claes, R. (2001). Proactieve Persoonlijkheidsschaal (PPS): Ontwikkeling en voorlopige validatie [Proactive Personality Scale (PPS): Development and preliminary validation]. Gedrag en Organisatie, 14, 291-304. Recuperado de https:// psycnet.apa.org/record/2001-05434-003

Salessi, S., \& Omar, A. (2016). Satisfacción laboral genérica. Propiedades psicométricas de una escala para medirla. Revista Alternativas en Psicología, 34, 93-108. Recuperado de http://alternativas.me/23-numero-34-febrero-julio-2016/116-satisfaccion-laboral

Salessi, S., \& Omar, A. (2018a). Comportamientos proactivos en el trabajo: validación y análisis psicométrico de una escala. Actualidades en Psicología, 32(124), 33-49. doi: 10.15517/ap.v32i124.30642

Salessi, S., \& Omar, A. (2018b). Cuestionario de implicación en el trabajo. Propiedades psicométricas de su adaptación en Argentina. Revista Mexicana de Psicología, 35(2), 96-114. Recuperado de https://www. redalyc.org $/$ articulo.oa?id $=243059346007$
Seibert, S., Crant, J., \& Kraimer, M. (1999). Proactive personality and career success. Journal of Applied Psychology, 84, 416-427. doi: 10.1037//0021-9010.84.3.416

Spitzmuller, M., Sin, H., Howe, M., \& Fatimah, S. (2015). Investigating the uniqueness and usefulness of proactive personality in organizational research: A meta-analytic review. Human Performance, 28, 351379. doi: 10.1080/08959285.2015.1021041

Tabachnick, B. G., \& Fidell, L. S (2013). Using Multivariate Statistics (6th ed.). Boston, MA: Pearson.

Thomas, J., Whitman, D., \& Viswesvaran, C. (2010) Employee proactivity in organizations: A comparative meta-analysis of emergent proactive constructs. Journal of Occupational \& Organizational Psychology, 83, 275-300. doi: 10.1348/09637910X502359

Timmerman, M. E., \& Lorenzo-Seva, U. (2011). Dimensionality assessment of ordered polytomous items with parallel analysis. Psychological Methods, 16, 209-220. doi: 10.1037/a0023353

Trifiletti, E., Capozza, D., Pasin, A., \& Falvo, R. (2009). A validation of the Proactive Personality Scale. Testing, Psychometrics, Methodology in Applied Psychology, 16(2), 77-93. Recuperado de https://psycnet.apa. org/record/2009-11829-002

Tornau, K., \& Frese, M. (2013). Construct clean-up in proactivity research: A meta-analysis on the nomological net of work-related proactivity concepts and their incremental validities. Applied Psychology: An International Review, 62, 44-96. doi: 10.1111/j.1464-0597.2012.00514.

Zhou, L., \& Shi, Z. (2009). Psychometric properties of the Chinese translation of the Proactive Personality Scale. Psychological Reports, 105, 43-56. doi: 10.2466/PR0.105.1.43-56

Recebido em: 08/01/2018

Reformulado em: 15/04/2020

Aprovado em: 09/06/2020 
Sobre las autoras:

Solana Salessi - argentina. Doctora en Psicología por la Universidad Nacional de La Plata, Argentina. Se desempeña como investigadora asistente del del Consejo Nacional de Investigaciones Científicas y Técnicas (CONICET). Es profesora asociada en el Departamento de Cultura, Educación y Conocimiento de la Universidad Nacional de Rafaela, Argentina. Autora de artículos en revistas especializadas y un capítulo de libro por invitación.

ORCID: https://orcid.org/0000-0001-9496-9493.

E-mail: solanasalessi@gmail.com

Alicia Omar - argentina. Doctora en Psicología por la Universidad Nacional de San Luis, Argentina. Se desempeña como investigadora científica del Consejo Nacional de Investigaciones Científicas y Técnicas (CONICET). Es profesora invitada de universidades argentinas y extranjeras para dictar cursos y seminarios sobre metodología de la investigación y comportamiento organizacional en el marco de carreras de postgrado.

ORCID: https://orcid.org/0000-0001-6613-2565.

E-mail:agraomar@yahoo.com

Dirección postal:

Solana Salessi

Universidad Nacional de Rafaela, Bv. Roca 989, Rafaela (2000)

Santa Fe, Argentina

Tél: +540340415500180 